\title{
artigo
}

Cunha, R.P.; Correia, D.M.S.; Camacho, A.C.L.F.; Hipólito, R.L.; Ferreira, S.C.M.; Pinheiro, F.P.;

Quiz Online para estudantes de enfermagem sobre cateterismo enteral e suas dimensões na prática

\section{Quiz Online para estudantes de enfermagem sobre cateterismo enteral e suas dimensões}

\section{na prática}

\section{RESUMO}

Objetivo: identificar os índices de acertos e erros por graduandos de enfermagem quanto aos pontos de atualização sobre cateterismo enteral nas dimensões clínica, técnica e legal. Método: estudo descritivo-exploratório, tipo Survey, no período de 02 a 05 de julho de 2017, com 51 participantes por meio de um Quiz Online, elaborado com 36 questões distribuídas em dimensões clínica, técnica e legal, sendo estabelecido para cada questão um índice satisfatório de $\geq 70 \%$, e envio de todas as respostas baseadas em evidências científicas, permitindo a autoavaliação. Resultados: 0 índice satisfatório foi alcançado pelos graduandos em 7 questões $(58,3 \%)$, na dimensão clínica; enquanto na dimensão técnica foram 8 questões $(50 \%)$ sobre cuidados de enfermagem; e, por fim, somente em $3(37,5 \%)$ na dimensão legal. Conclusão: destaca-se a importância deste jogo educacional pela primeira vez no ensino de fundamentos, dada a significativa adesão dos graduandos, a atualização, a autoavaliação, e sobretudo dimensões para prática. DESCRITORES: Enfermagem; Tecnologia Educacional; Sondas de Alimentação Enteral.

\section{ABSTRACT}

Objective: identify the right and wrong indices of nursing undergraduates regarding the update points on enteral catheterization in the clinical, technical and legal dimensions. Method: a descriptive exploratory study, type Survey, from July 2 to 5, 2017, with 51 participants through an Online Quiz, prepared with 36 questions distributed into clinical, technical and legal dimensions, and a question was established for each question. satisfactory rate of $\geq 70 \%$, and submission of all answers based on scientific evidence, allowing self-assessment. Results: the satisfactory index was reached by the undergraduates in 7 questions $(58.3 \%)$, in the clinical dimension; while in the technical dimension there were 8 questions (50\%) about nursing care; and finally, only $3(37.5 \%)$ in the legal dimension. Conclusion: The importance of this educational game for the first time in the teaching of fundamentals is highlighted, given the significant adhesion of the undergraduates, the updating, the self-assessment, and especially the dimensions for practice.

KEYWORDS: Nursing; Educational Technology; Enteral Feeding Probes.

\section{RESUMEN}

Objetivo: identificar los índices correctos e incorrectos de los estudiantes de pregrado en enfermería con respecto a los puntos de actualización sobre el cateterismo enteral en las dimensiones clínica, técnica y legal. Método: estudio exploratorio descriptivo, tipo Encuesta, del 2 al 5 de julio de 2017, con 51 participantes a través de un cuestionario en línea, preparado con 36 preguntas distribuidas en dimensiones clínicas, técnicas y legales, que se establecen para cada pregunta. tasa satisfactoria de $\geq 70 \%$ y presentación de todas las respuestas basadas en evidencia científica, lo que permite la autoevaluación. Resultados: los estudiantes de pregrado alcanzaron un índice satisfactorio en 7 preguntas (58,3\%), en la dimensión clínica; mientras que en la dimensión técnica hubo 8 preguntas (50\%) sobre cuidados de enfermería; y finalmente solo 3 (37.5\%) en la dimensión legal. Conclusión: Se destaca la importancia de este juego educativo por primera vez en la enseñanza de los fundamentos, dada la importante adhesión de los estudiantes de pregrado, la actualización, la autoevaluación y especialmente las dimensiones para la práctica.

PALABRAS CLAVE: Enfermería; Tecnología Educativa; Sondas de Alimentación Enteral.

RECEBIDO EM: 10/11/2019 APROVADO EM: 11/11/2019

\section{Rodrigo Pernas da Cunha}

Mestrando em Pesquisa Clínica em Doenças Infecciosas na Fundação Oswaldo Cruz.

\section{Dayse Mary da Silva Correia}

Doutora em Ciências Cardiovasculares. Professor Adjunto da Escola de Enfermagem Aurora de Afonso Costa da Universidade Federal Fluminense. 


\section{Alessandra Conceição L. Funchal Camacho}

Doutor em Enfermagem. Professor da Escola de Enfermagem Aurora de Afonso Costa da Universidade Federal Fluminense.

\section{Rodrigo Leite Hipólito}

Doutor em Enfermagem. Professor da Escola de Enfermagem Aurora de Afonso Costa da Universidade Federal Fluminense.

\section{Simone Cruz Machado Ferreira}

Doutor em Enfermagem. Professor da Escola de Enfermagem Aurora de Afonso Costa da Universidade Federal Fluminense.

\section{Fernanda Machado Pinheiro}

Doutoranda do Programa do Programa de Pós-graduação em Ciências do Cuidado em Saúde da Escola de Enfermagem Aurora de Afonso Costa da Universidade Federal Fluminense.

\section{INTRODUÇÃO}

0 jogo educativo é uma eficiente estratégica pedagógica no ensino-aprendizagem, na comunicação e expressão, bem como visa proporcionar satisfação imediata aos participantes $^{(1)}$. Visto que o perfil dos alunos e professores, quanto aos métodos e instrumentos de ensino e às abordagens pedagógicas utilizadas passaram por influências globais, havendo um aumento na participação ativa dos estudantes no processo de aprendizagem, na otimização do rendimento acadêmico e no ambiente educacional ${ }^{(2)}$.

A disponibilidade e o crescente progresso na produção de hardwares e softwares têm criado oportunidades para o avanço no ensino, e para o de enfermagem não tem sido diferente. Sendo o objetivo desses sistemas computacionais o de auxiliar e aumentar as habilidades do usuário para troca de informações com o computador e preparar os estudantes para a nova realidade tecnológica ${ }^{(3)}$. Além da relação dessas iniciativas didáticas nos Cursos de Graduação de Enfermagem contribuírem para reorientação durante a formação dos profissionais em consonância com o Sistema Único de Saúde - SUS ${ }^{(1)}$.

De modo específico e de caráter transversal, a disciplina de Fundamentos de Enfermagem na instituição visa contribuir na formação com princípios básicos e de média complexidade e no desenvolvimento de habilidades a serem aplicados de modo permanente ao longo da formação e da atuação, bem como deve proporcionar atualização de temas relevantes no cuidado em saúde do paciente hospitalizado.

Diante disso, durante o ensino de Fundamentos de Enfermagem, no ano de 2016, foi proposto um trabalho por meio do desenvolvimento de trabalho de monitoria, um jogo educacional, para o qual utilizou-se um Quiz online, tipo Survey, com 20 perguntas sobre aspectos das técnicas para inserção da sonda nasogástrica e da sonda nasoenteral, e no qual participaram de modo voluntário de 64 graduandos. Logo, ficou estabelecida uma perspectiva de aperfeiçoamento, pois $54,7 \%$ dos graduandos respondeu nas primeiras 24 horas, $98,4 \%$ apontou ser um dispositivo tecnológico muito importante, além de ter havido uma grande interação proporcionada pelo jogo. Portanto, contribuindo como uma promissora estratégia para o ensino de temas relevantes e Práticas Baseadas em Evidências (PBE) para disciplina de Fundamentos de Enfermagem ${ }^{(4)}$.

Dessa forma, a partir da importância na utilização de um Quiz, da necessidade de uma versão mais abrangente sobre a sonda nasoenteral, da aproximação de um meio acessível de atualização aos graduandos e dada a atuação da equipe de enfermagem na Terapia Nutricional, fez-se como questão norteadora Qual o entendimento dos graduandos sobre as dimensões clínica, técnica e legal do cateterismo enteral? E para tal, propóe-se identificar os índices de acertos e erros por graduandos de enfermagem quanto aos pontos de atualização sobre cateterismo enteral nas dimensões clínica, técnica e legal.

\section{METODOLOGIA}

O estudo foi do tipo descritivo-exploratório, por meio de um Survey ${ }^{(5)}$, com graduandos da Escola de Enfermagem Aurora de Afonso Costa, da Universidade Federal Fluminense (EEAAC/UFF) no Estado do Rio de Janeiro.

A amostra constituída foi não-probabilística composta por 51 acadêmicos de enfermagem do $4^{\circ}$ ao $9^{\circ}$ período do referido Curso de Graduação. E os critérios de inclusão utilizados foram: participação voluntária, acesso ao ambiente digital por meio do telefone, tablet ou computador e concordância digital do Termo de Consentimento Livre e Esclarecido (TCLE).

A coleta de dados foi realizada entre os dias 02 a 05 de agosto de 2017, utilizando um Quiz Online, e para o qual um link de acesso foi enviado aos participantes, contendo dados de identificação, bem como o TCLE no formato digital.

O referido Quiz foi elaborado na plataforma Google Forms para esta pesquisa no formato de distribuição de 36 questões por 03 (três) dimensões referentes ao tema do cateterismo nasoenteral, a saber: clínica, contendo 12 (doze) perguntas sobre segurança do paciente, indicações clínicas, tempo de permanência das sondas; técnica, com 16 perguntas direcionadas à mensuração, aos testes de localização após inserção, e cuidados de enfermagem; e legal com 08 perguntas sobre os aspectos do Código de Ética dos Profissionais de Enfermagem (CEPE) e do Código Penal Brasileiro (CPB).

Cabe salientar que, no Quiz, havia 


\section{artigo}

Cunha, R.P.; Correia, D.M.S.; Camacho, A.C.L.F.; Hipólito, R.L.; Ferreira, S.C.M.; Pinheiro, F.P.;

Quiz Online para estudantes de enfermagem sobre cateterismo enteral e suas dimensões na prática

um espaço livre para pontos positivos, negativos e sugestões, bem como todas as respostas com base em evidência científica foram enviadas imediatamente após o preenchimento final, permitindo aos participantes a autoavaliação, e o índice satisfatório de acertos estabelecido para cada questão foi de $\geq 70 \%$.

Os resultados foram obtidos na referida plataforma, expressos em percentual (\%) para os dados de identificação e para todas as questões (erros ou acertos).

A pesquisa foi submetida e aprovada em 2017 no Comitê de Ética em Pesquisa da Faculdade de Medicina do Hospital Universitário Antônio Pedro (CEP/ FMHUAP), sob o Parecer n. ${ }^{\circ}$ 2.140.945.

\section{RESULTADOS}

Os dados de identificação apontaram que dos 51 participantes, 41 graduandos de enfermagem eram do sexo feminino $(80,4 \%)$. Quanto à idade dos graduandos, observou-se uma maior frequência entre 27 graduandos (52,9\%) na faixa etária de 21 a 23 anos, seguida de 29,4\% (15) de 24 a 26 anos, sendo 08 acima de 27 anos (15,7\%), e $2 \%$ de 18 a 20 anos. Além de ter havido alta prevalência $(80,4 \%)$ de respostas ao Quiz de graduandos do $7^{\circ}, 8^{\circ}$ e $9^{\circ}$ períodos do referido Curso de Graduação.

Quanto à dimensão clínica, no Quadro 1 , observa-se que das 12 questôes, os graduandos tiveram o alcance de acertos esperados em $58,3 \%$ (7), as quais se referiam a diagnóstico clínico, sinais clínicos, tempo de permanência da sonda e cuidado de enfermagem para com a velocidade de infusão e permeabilidade da sonda. E quanto ao baixo índice de acertos bem como erros, $41,7 \%$ (5), estavam direcionados à terminologia e às complicações.

Quando analisadas as 16 questôes da dimensão técnica, observa-se no Quadro 2 que em $50 \%$ (8) das questões, os graduandos obtiveram acertos referentes a algumas perguntas sobre o fio-guia, o Raio-X e cuidados de enfermagem. Enquanto, para igual percentual, foram identificados erros quanto à cuidados de enfermagem (posicionamento do paciente, banho no leito e conservação do frasco

Quadro 1. Resultado Global do Quiz sob a dimensão clínica. Niterói, RJ, Brasil, 2017.

\begin{tabular}{|c|c|c|c|c|}
\hline \multirow{2}{*}{ QUESTÕES } & \multicolumn{2}{|c|}{ ACERTOS } & \multicolumn{2}{|c|}{ ERROS } \\
\hline & (N) & (\%) & (N) & (\%) \\
\hline 1- Qual o significado de Índice de Quetelet? & 28 & 54,9 & 23 & 45,1 \\
\hline $\begin{array}{l}\text { 2- Em um paciente internado com Glasgow 8, entubado por via nasotraqueal e instável } \\
\text { hemodinamicamente, a dieta enteral está indicada ou contra-indicada? }\end{array}$ & 23 & 45,1 & 28 & 54,9 \\
\hline 3- Qual o tipo de complicação, na nutrição enteral, considerada de maior gravidade? & 34 & 66,7 & 17 & 33,3 \\
\hline $\begin{array}{l}\text { 4- A sonda enteral pode sofrer com ação de secreções digestivas trazendo danos ao } \\
\text { paciente? }\end{array}$ & 28 & 54,9 & 23 & 45,5 \\
\hline $\begin{array}{l}\text { 5- Durante a administração de nutrição enteral, qual(is) complicação(ões) podem ser obser- } \\
\text { vadas? }\end{array}$ & 34 & 66,7 & 17 & 33,3 \\
\hline 6- O que significa Estase Gástrica durante o uso da terapia de Nutrição Enteral? & 46 & 90,2 & 5 & 9,8 \\
\hline $\begin{array}{l}\text { 7-Para a instalação da sonda nasoenteral no intestino delgado o que é necessário no } \\
\text { processo digestivo? }\end{array}$ & 36 & 70,6 & 15 & 29,4 \\
\hline $\begin{array}{l}\text { 8- Quais os sinais clíncos apresentados pelo paciente, que podem indicar um falso trajeto } \\
\text { de uma sonda naoenteral? }\end{array}$ & 38 & 74,5 & 13 & 25,5 \\
\hline $\begin{array}{l}\text { 9- A previsão de uso de sonda saso ou oroenteral é de um curto perído de tempo ( } 3 \text { a } 4 \text { se- } \\
\text { manas). Quais procedimentos estã indicados quando há uma previsão acima deste tempo? }\end{array}$ & 37 & 72,5 & 14 & 27,5 \\
\hline 10- Porque a velocidade de infusão da dieta deve ser controlada por bomba de infusão? & 39 & 76,5 & 12 & 23,5 \\
\hline $\begin{array}{l}\text { 11- Por que se de deve administrar água na sonda naso ou otoenteral antes e depois de } \\
\text { administração de medicamento e/ou dieta? }\end{array}$ & 44 & 86,3 & 7 & 13,7 \\
\hline $\begin{array}{l}\text { 12- Um paciente em uso de sonta naenteral com prescrição de dois medicamnetos em } \\
\text { apresentação de compridos através da sonda, no mesmo horário. Qual a conduta correta? }\end{array}$ & 38 & 74,5 & 13 & 25,5 \\
\hline
\end{tabular}

Quadro 2. Resultado Global do Quiz sob a dimensão técnica. Niterói, RJ, Brasil, 2017.

\section{QUESTÕES}

1- Para facilitar a migração de uma sonda nasoenteral para a posição pós-pilórica é recomendado o posicionamento do paciente em qual decúbito?

\begin{tabular}{|c|c|c|c|}
\hline \multicolumn{2}{|c|}{ ACERTOS } & \multicolumn{2}{c|}{ ERROS } \\
\hline (N) & (\%) & (N) & (\%) \\
\hline 24 & 47,1 & 27 & 52,9 \\
\hline
\end{tabular}




\begin{tabular}{|c|c|c|c|c|}
\hline $\begin{array}{l}\text { 2- Por que não se deve recolocar o fio-guia da sonda nasoenteral já posicionada no pacien- } \\
\text { te na tentativa de desobstrução? }\end{array}$ & 43 & 84,3 & 8 & 15,7 \\
\hline $\begin{array}{l}\text { 3- Qual o tempo recomendado para um paciente permanecer em uso da sonda nasoente- } \\
\text { ral? }\end{array}$ & 35 & 68,6 & 16 & 31,4 \\
\hline $\begin{array}{l}\text { 4- Para a realização de um banho no leito do paciente, quanto tempo antes recomenda-se } \\
\text { interromper a administração da dieta? }\end{array}$ & 25 & 49 & 26 & 51 \\
\hline $\begin{array}{l}\text { 5- Qual o período de tempo após a passagem de uma sonda nasoenteral recomenda-se a } \\
\text { realização do exame de Raio X para verificação do poscionamento da sonda? }\end{array}$ & 4 & 7,8 & 47 & 92,2 \\
\hline $\begin{array}{l}\text { 6- Qual cuidado de enfermafem deve ser realizado para prevenir a obstrução de uma } \\
\text { sonda? }\end{array}$ & 39 & 76,5 & 12 & 25,5 \\
\hline $\begin{array}{l}\text { 7- Quando se decide por adiar a administração da dieta enteral, como recomenda se con- } \\
\text { servar o frasco (fechado) até seu uso em } 24 h \text { ? }\end{array}$ & 24 & 47,1 & 27 & 52,9 \\
\hline $\begin{array}{l}\text { 8- Após a abertura do frasco de nutrição enteral manipulada, qual tempo de validade do } \\
\text { conteúdo? }\end{array}$ & 18 & 35,3 & 33 & 64,7 \\
\hline $\begin{array}{l}\text { 9- Quais dos testes abaixo podem ser utilizados para verificar a localização de uma sonda } \\
\text { nasoenteral? }\end{array}$ & 35 & 68,6 & 16 & 31,4 \\
\hline $\begin{array}{l}\text { 10- Durante a inserção de uma sonda nasoenteral, o paciente pode "ajudar" o profissional } \\
\text { durante a passagem, realizando qual ação? }\end{array}$ & 47 & 92,2 & 4 & 7,8 \\
\hline $\begin{array}{l}\text { 11- Qual a recomendação da retirada do fio-guia após a inserção de uma sonda nasoen- } \\
\text { teral? }\end{array}$ & 46 & 90,2 & 5 & 9,8 \\
\hline $\begin{array}{l}\text { 12- Para qual dessas sondas é ndicado a realização do exame de Raio X para verificar sua } \\
\text { localização? }\end{array}$ & 46 & 84,3 & 8 & 15,7 \\
\hline 13- Para que ser o fio-guia a sonda nasoenteral? & 38 & 74,5 & 13 & 25,5 \\
\hline $\begin{array}{l}\text { 14- Sobre o uso do EPI durante a realização de uma passagem de sonda nasoenteral, a } \\
\text { luva poderá ser. }\end{array}$ & 34 & 66,7 & 17 & 33,3 \\
\hline 15- Qual das opções abaixo é uma função primordial da sonda nasoenteral? & 43 & 84,3 & 8 & 15,7 \\
\hline $\begin{array}{l}\text { 16- Para o posicionamento de uma sonda enteral na posição jejunal, qual a medida de } \\
\text { introdução que deva ser calculada? }\end{array}$ & 27 & 52,9 & 24 & 47,1 \\
\hline
\end{tabular}

da dieta), permanência da sonda e indicação do Raio-X.

E por fim, analisando a dimensão legal, no Quadro 3, foi observado que em
5 questões $(62,5 \%)$, os graduandos não atingiram o esperado de acertos sobre aspectos do Código de Ética de Enfermagem $^{(6)}$, atuação da Enfermagem na
Terapia Nutricional segundo o Conselho Federal de Enfermagem - $\operatorname{COFEN}^{(7)}$ e Agência Nacional de Vigilância Sanitária - ANVISA ${ }^{(8)}$.

\section{Quadro 3. Resultado Global do Quiz pela dimensão legal. Niterói, RJ, Brasil, 2017.}

\section{QUESTÕES}

1- A qual profissional de saúde está atribuída a responsabilidade pela conservação da nutrição enteral após o recebimento na enfermaria?

2- Qual profissional é responsável por "estabelecer o acesso enteral, por via oro/nasogástrica ou tranpilórica, para administração da Nutrição Enteral"?

3- Qual orgão refulamenta a atuação de equipe de enfermagem em Terapia Nutricional?

4- Qual é a Resolusão do COFEN que regulamente a atuação da equipe de enfermagem em Terapia Nutricional?

5- 0 enfermeiro(a) com desprepara tecnico e, causando dano ao paciente durante a inserção de uma sonda nasoenteral, estaria cometendo ato de:

6- Ao administrar um frasco de dieta, o enfermeiro por desateção, trocou o frasco de um paciente para o outro, logo a infração foi caraterizada como:

\begin{tabular}{|c|c|c|c|c|}
\hline \multicolumn{2}{|c|}{ ACERTOS } & \multicolumn{2}{c|}{ ERROS } \\
\cline { 2 - 5 }$(\mathbf{N})$ & $(\%)$ & (N) & $(\%)$ \\
\hline 40 & 78,4 & 11 & 21,6 \\
\hline 48 & 94,1 & 3 & 5,9 \\
\hline 11 & 21,6 & 40 & 78,4 \\
\hline 20 & 39,2 & 31 & 60,8 \\
\hline 21 & 41,2 & 30 & 58,8 \\
\hline
\end{tabular}




\section{artigo}

Cunha, R.P.; Correia, D.M.S.; Camacho, A.C.L.F.; Hipólito, R.L.; Ferreira, S.C.M.; Pinheiro, F.P.;

Quiz Online para estudantes de enfermagem sobre cateterismo enteral e suas dimensões na prática

7- Um técnico de enfermagem durante o plantão, o qual está sob a supervisão do enfermeiro, conectou o equipo da dieta enteral no acesso venoso do paciente, o qual veio à óbtito. Quais são os atos imediatos identificados diante desta infração gravíssima do Código de Ética dos profissionais de Enfermagem para o técnico e enfermeiro, respectivamente?

8- Qual RDC da ANVISA identifica como responsável o enfermeiro de "proceder ou assegurar a colocação da sonda oro/nasogástrica ou transpilórica"?

\begin{tabular}{|l|l|l|l|}
\hline 16 & 31,4 & 35 & 68,6 \\
\hline 25 & 49 & 26 & 51 \\
\hline
\end{tabular}

\section{DISCUSSÃO}

É a Terapia Nutricional no ambiente hospitalar uma atividade multiprofissional, sobretudo com a participação do Enfermeiro exercendo competências em níveis clínico, técnico e legal( ${ }^{(8)}$.

Neste estudo, cabe salientar no perfil dos graduandos quanto a maioria ser do sexo feminino e a faixa etária entre 21 a 23 anos, corrobora com outros estudos ${ }^{(9,10)}$. Quanto aos períodos dos participantes, observamos que a maioria dos participantes foi dos últimos períodos, podendo-se inferir um grande interesse, principalmente de atualização.

Quando da dimensão clínica, a qual buscou pontos relevantes da segurança do paciente, indicações clínicas e tempo de permanência das sondas, foi a que apresentou melhor desempenho dos graduandos. Haja visto que diagnóstico clínico, sinais clínicos, tempo de permanência da sonda e cuidado de enfermagem para com a velocidade de infusão e permeabilidade da sonda foram questões com um índice satisfatório de acertos.

É sabido que, durante o uso de Terapia de Nutrição Enteral, o enfermeiro deve estar atento à condição clínica de gastroparesia. Ou seja, há uma diminuição da força contrátil do estômago, ocorrendo um esvaziamento gástrico retardado - estase gástrica ${ }^{(11)}$.

E que a instalação da sonda no intestino ocorre de forma lenta, dependendo da peristalse gastrintestinal, o que permitirá sua passagem pelo piloro. Sendo a tosse, dificuldade respiratória, cianose e agita- ção, os principais sinais apresentados pelo paciente durante um falso trajeto na passagem de uma sonda nasoenteral ${ }^{(12)}$.

Quanto ao suporte nutricional prolongado, é indicada a gastrostomia quando não há risco de aspiração e; a jejunostomia, quando há risco de aspiração ${ }^{(13)}$.

A diarreia é a complicação mais comum na alimentação por sonda e, geralmente, resulta de infusão rápida. Portanto, o uso da bomba de infusão com gotejamento controlado é recomendado ${ }^{(14)}$ dentro do cuidado de enfermagem.

E por fim, para evitar a ocorrência de obstrução da sonda entera ${ }^{1(15)}$, a administração de água filtrada ( 5 a $10 \mathrm{ml}$ ) durante a assistência de enfermagem antes e após a administração de medicamentos ou dieta é imprescindível. E quando houver dois ou mais medicamentos a serem administrados por sonda no mesmo horário, triturar, diluir e infundir cada um separadamente. Nunca misturar ou administrar medicamentos concomitantemente no mesmo recipiente, evitando obstrução do acesso por precipitação, além de interação medicamentosa ${ }^{(16)}$.

Entretanto, de modo insatisfatório, ou seja, abaixo de $70 \%$ de acertos ficaram direcionados à terminologia e às complicações, onde o Índice de Massa Corporal, denominado IMC ou índice de Quetelet é um método para avaliação do acúmulo de gordura, correlacionando o peso $(\mathrm{Kg})$ e altura - $\mathrm{m} 2^{(12)}$.

E foi esclarecido que as complicações da terapia nutricional enteral (TNE) variam de acordo com o serviço hospitalar, porém as mais frequen- tes relacionadas na literatura científica podem ser divididas em quatro grupos: digestivas (diarreia, vômitos etc.), mecânicas (como a perda da sonda enteral), infecciosas (por contaminação da dieta enteral) e de ordem operacional - como os atrasos na administração da dieta enteral ${ }^{(17)}$. Alguns autores mencionam ainda complicações metabólicas e psicológicas com o uso da TNE. Sendo a broncoaspiração, responsável por complicação respiratória grave $\mathrm{e}^{(14)}$.

Quanto à indicação ao paciente grave, a terapia nutricional enteral está indicada precocemente no paciente grave (entre 24 e 48 horas após a admissão), desde que o paciente esteja hemodinamicamente estável(18).

E ainda que, não há evidências de que haja degradação da sonda pelas secreções digestivas do paciente, pois as sondas para nutrição enteral devem ser feitas de borracha de silicone, poliuretano ou de outros polímeros, flexíveis, de fino calibre, leves, macias, resistentes à ação das secreções digestivas e de alta tolerabilidade por parte dos pacientes biocompatível(12).

Ao avaliar a dimensão técnica, a qual teve foco em mensuração e aos testes de localização após inserção da sonda, e cuidados de enfermagem, percebe-se um percentual equilibrado entre os graduandos tanto para satisfatório quanto insatisfatório.

Coube lembrar que quando da possibilidade de posicionamento do paciente para facilitar a migração da sonda, recomende-se que o paciente deve ser posicionado em decúbito lateral direito ${ }^{(18)}$, pois o posicionamento no intestino ocorre de for- 
ma lenta, dependendo da peristalse gastrintestinal; que o fio-guia não deva ser reintroduzido na sonda na tentativa de desobstrui-la, porque poderá perfurá-la e lesar a mucosa esofagiana ${ }^{(12)}$, sondas com inserção transnasal ou oral são recomendadas por curto período de tempo, com uma duração até de 3 a 4 semanas ${ }^{(12)}$.

E ainda que diante do banho no leito, recomenda-se interromper a administração de dieta enteral, pelo menos 30 minutos antes de realizar qualquer procedimento que envolva mudança de decúbito ou quando se precisa manter a cabeceira baixa ${ }^{(18)}$; e que a literatura recomenda aguardar pelo menos 3 horas após a passagem da sonda enteral para se realizar o exame de Raio X, pois este é o tempo médio em que a peristalse e a gravidade irão atuar em conjunto durante a migração da sonda pelo canal digestivo, do estômago até o intestino delgado, tendo sua posição final pós-pilórica ${ }^{(18)}$; e reforça-se que a administração da água em intervalos concomitantes à administração da dieta e medicamentos, sendo estes momentos antes e após a administração da dieta enteral ${ }^{(13)}$.

As dietas manipuladas possuem validade de até 24 horas após a abertura da embalagem, se guardadas em geladeira ${ }^{(18)}$, sempre apropriada para medicamentos/ nutrição, quando a sua instalação for postergada.

O Raio-X a beiro do leito de abdome é considerado o padrão-ouro para testar e garantir o posicionamento correto da sonda de poliuretano e a retirada do fio metálico e flexível (fio-guia), o qual destina-se facilitar a introdução nasal ${ }^{(18)}$. Mas, não excluindo o auxílio de outros testes de localização, como a ausculta e a medição de $\mathrm{pH}^{(11)}$, em acordo com o protocolo institucional. E durante o procedimento, avaliar a possibilidade de ajuda do paciente, o qual pode ser orientado a relaxar os músculos da face e, quando sentir que a sonda

\section{O estudo reforça}

a importância do

uso deste jogo

educacional no ensino

de Fundamentos

de Enfermagem,

pois foi observado

que o Quiz online,

como estratégia

para a construção

de conhecimento,

apresentou uma

significativa adesão

à participação de

maneira voluntária,

identificando, assim,

a importância do

ensino nas dimensões

clínica, técnica e

legal aos alunos de

Fundamentos de

Enfermagem. chegou à garganta, orientá-lo para inspirar e engolir para evitar e sensação de náusea causada pela presença da sonda na faringe ${ }^{(11)}$.

Diante da necessidade do uso de equipamento de proteção individual (EPI), a luva de proteção pode ser não estéril (procedimento), tendo em vista que o procedimento não é considerado estéril e sim, limpo ${ }^{(18)}$.

E por fim, a medida calculada para introdução de uma sonda enteral para posicionamento jejunal é recomendada da ponta do nariz ao lóbulo da orelha e, deste até o apêndice xifoide, acrescentando de 10 a $15 \mathrm{~cm}$ - aproximadamente na altura da cicatriz umbilical ${ }^{(18)}$.

Os pontos de atualização para a dimensão legal foram destacados a partir de aspectos do $\mathrm{CEPE}^{(6)}$, atuação da Enfermagem na Terapia Nutricional segundo $\mathrm{COFEN}^{(7)}$ e ANVISA $^{(8)}$, e foi observado um elevado índice insatisfatório referente às questões.

Houve clareza de que na unidade o enfermeiro é responsável pela conservação da nutrição enteral e pela sua administração. E que cabe ao Enfermeiro estabelecer o acesso enteral, por via oro/nasogástrica ou transpilórica, para administração da NE, conforme procedimento pré-estabelecido.

Entretanto, não apresentaram um bom conhecimento sobre a resolução regulatória do $\operatorname{COFEN}^{(7)}$ e da $\mathrm{AN}$ VISA $^{(8)}$, ambas direcionadas a assegurarem, respectivamente, a atuação da equipe de enfermagem e identificando o enfermeiro como responsável de "proceder ou assegurar a colocação da sonda oro/nasogástrica ou transpilórica" ${ }^{(8)}$.

Ademais, sobre a aplicabilidade de conceitos básicos sobre implicações legais que podem envolver um procedimento de enfermagem, como a imperícia, negligência ou a imprudência ${ }^{(6)}$.

A construção de um Quiz Online baseado em dimensões proporcionou 


\section{artigo}

Cunha, R.P.; Correia, D.M.S.; Camacho, A.C.L.F.; Hipólito, R.L.; Ferreira, S.C.M.; Pinheiro, F.P.

ao graduando autoavaliação e uma visão abrangente do tema proposto.

\section{CONCLUSÃO}

O estudo reforça a importância do uso deste jogo educacional no ensino de Fundamentos de Enfermagem, pois foi observado que o Quiz online, como estratégia para a construção de conhecimento, apresentou uma significativa adesão à participação de maneira voluntária, identificando, assim, a importância do ensino nas dimensões clínica, técnica e legal aos alunos de Fundamentos de Enfermagem.
Neste contexto, o elevado quantitativo de respostas corretas referentes às dimensões, reforça a importância do processo de ensino-teórico e prático baseado em evidência científica para que se possam reproduzir práticas de assistência de alta qualidade.

\section{REFERÊNCIAS}

1. Gurgel SS, Taveira GP, Matias EO, Pinheiro PNC, Vieira NFC, Lima FET. Jogos educativos: recursos didáticos utilizados na monitoria de educação em saúde. REME - Rev Min Enferm [Internet]. 2017[citado em 18 de abr de 2019]; 21:e-1016. Disponivel em: http://www.reme.org.br/artigo/detalhes/1152.

2. Bezerril MS, Chiavone FBT, Lima JVH, Vitor AF, Ferreira Júnior MA, Santos VEP. Nursing education: a conceptual analysis of the evolutionary method of Rodgers. Escola Anna Nery [Internet]. 2018 [citado 17 de abr de 2019]; 22(4). Disponível em: http://www.scielo.br/scielo.php?script=sci_arttext\&pi$\mathrm{d}=$ S1414-81452018000400801\&lng=en\&tIng=en.

3. Lorenzetti J, Trindade LL, Pires DEP, Ramos FRS. Tecnologia, inovação tecnológica e saúde: uma reflexão necessária. Texto Contexto Enferm [Internet]. 2012 [citado 17 de abr de 2019]; 21(2):432-9. Disponivel em: http://www.scielo.br/pdf/tce/ v21n2/a23v21n2.pdf.

4. Cunha RP, Correia DMS. QUIZ virtual: uma estratégia educativa e interativa de atualização de Fundamentos de Enfermagem. Anais $23^{\circ}$ Pesquisando em Enfermagem - UFRJ [Internet]. Rio de Janeiro. 2016 [acesso em 18 abr 2016]. 169 p. Disponivel em: http://www.pesquisandoenfermagem.com.br/wpcontent/uploads/2016/05/anais_2016_pesquisando.pdf.

5. Jancew CC, Cendon BV, Ameno N. Websurveys como método de pesquisa. Informação \& Informação [Internet]. 2014 [citado 17 de abr de 2019]; 19(3):192-218. Disponivel em: http://www. uel.br/revistas/uel/index.php/informacao/article/view/17641/ pdf_42.

6. Conselho Federal de Enfermagem. Resolução Cofen n. ${ }^{\circ}$ 564/2017. Aprova o novo Código de Ética de Enfermagem [Internet]. Rio de Janeiro [citado 17 de abr de 2019]. Disponivel em: http://www.cofen.gov.br/resolucao-cofen-no-5642017_59145. html.

7. Conselho Federal de Enfermagem. Resolução Cofen n. 453/2014. Aprova a Norma Técnica que dispõe sobre a Atuação da Equipe de Enfermagem em Terapia Nutricional [Internet]. Rio de Janeiro [citado 17 de abr de 2019] Disponivel em: http://www. cofen.gov.br/resolucao-cofen-no-04532014_23430.html.

8. Agência Nacional de Vigilância Sanitária (ANVISA). Resolução - RDC n. ${ }^{\circ}$ 63/ 2000. Regulamento Técnico para a Terapia de Nutrição Enteral [Internet]. Brasília [citado 17 de abril de 2019] Disponivel em :http://bvsms.saude.gov.br/bvs/saudelegis/anvisa/2000/rdc0063_06_07_2000.html.

9. Pascon DM, Otrenti E, Mira VL. Percepção e desempenho de graduandos de enfermagem em avaliação de metodologias ativas. Acta Paul Enferm [Internet]. 2018 [citado 17 de abr de 2019]; 31(1):61-70. Disponivel em : http://www.scielo.br/pdf/ ape/v31n1/0103-2100-ape-31-01-0061.pdf.

10. Freire ACS, Vasconcelos HCA. Doação de sangue: conhecimento, prática e atitude de acadêmicos de enfermagem de uma instituição do interior do Ceará. REME- Rev Min Enferm [Internet]. 2013 [citado 17 de abr de 2019]; 17(2): 296-311. Disponível em: http://www.reme.org.br/artigo/detalhes/651.

11.Teixeira ACC, Caruso L, Soriano FC. Terapia nutricional enteral em unidade de terapia intensiva: infusão versus necessidades. Rev Bras Ter Int [Internet]. 2006 [citado 17 de abril de 2019]; 18(4):331-337. Disponivel em: http://rbti.org.br/artigo/detaIhes/0103507X-18-4-3.

12. Unamuno MRDL, Marchinil JS. Sonda nasogástrica/nasoentérica: cuidados na instalação, na administração da dieta e prevenção de complicações. Ribeirão Preto [Internet]. 2015 [acesso 01 jul 2017]; 35(02):95-101. Disponivel em: http://www.proac. uff.br/esai2/sites/default/files/sonda_nasogastrica_artigo. pdf.

13. Faculdade de Medicina de Marília. Protocolo de terapia nutricional enteral em adultos. Marília [Internet]. 2012 [acesso em 01 jul 2017]; 1-25. Disponivel em: https://www.famema.br/institucional/emtn/doc/Protocolo\%20de\%20Terapia\%20Nutricional\%20 Enteral\%20-\%20Adulto.pdf.

14. Gimenez FRE, Reis RK. Manuseio de sonda enteral: uma revisão integrativa da literatura. São Paulo [Internet]. 2015 [acesso em 01 jul 2017]; 17(97):13-9. Disponivel em: http://www.researchgate.net/publication/279884916_Manuseio_de_sonda_enteral_uma_reviso_integrativa_da_literatura.

15. Júnior JAM, Santos OF, Gomes MRL, Cabral LAF, Pereira PL. Protocolo de terapia nutricional e parenteral. Teresina [Internet]. 2012 [acesso em 01 jul 2017]; 4-17 p. Disponivel em: http:// www.hgv.pi.gov.br/download/201204/HGV25_acf79f701f.pdf.

16. Ribeiro PC, et al. Manual para administração de medicamentos por acessos enterais. São Paulo: Editora Atheneu; 2014. 448p.

17. Coppini LZ, Waitzberg DL. Complicações em nutrição enteral. In: Nutrição Oral Enteral e Parenteral na Prática Clínica. 4. Ed. ed. São Paulo, Atheneu; 2009. 907-917p.

18. Associação Médica Brasileira. Projeto Diretrizes. Volume IX. Brasília, DF: Conselho Federal de Medicina; 2011. 494p. 